\title{
PLANTAR SURFACE CONTOURS USING CLOSE RANGE PHOTOGRAMMETRY
}

\author{
${ }^{1,2}$ Jasmin Al-Baghdadi, ${ }^{1}$ Albert Chong, ${ }^{1}$ Duaa Alshadli, ${ }^{3}$ Richard Newsham-West and ${ }^{3}$ Peter \\ Milburn \\ ${ }^{1}$ University of Southern Queensland, Toowoomba, Australia. \\ ${ }^{2}$ Technical College, Baghdad, Iraq. \\ ${ }^{3}$ Griffith University, Gold Coast, Australia. email contact: p.milburn@griffith.edu.au
}

\begin{abstract}
Introduction
Motion of the foot during foot strike (contact), stance (midstance) and toe-off (propulsive phases) is of particular interest to clinicians as it provides information on the internal loading of the foot and the physical interaction between the foot and the surface. However, to capture video footage of this interaction, it is necessary to image the event through a clear substrate. However, images suffer from refraction distortion, and details of the methods used to calculate the glass refraction distortion correction can be found in $\mathrm{Al}$ Baghdadi et al. (2013). The same techniques are exploited to investigate the plantar surface of the foot on a glass-topped AMTI force plate system during the stance phase of the gait cycle.
\end{abstract}

\section{Purpose of the study}

The purpose of this pilot study was to implement a multi-camcorder system to capture images of the plantar surface through a glass-topped force plate. This enabled the loading on the contact-surface to be synchronised with the 3D surface contours.

\section{Methods}

Four JVC Everio GZ-HD500S HD camcorders (pixel count (still and video modes) = 1920x 1080; range of the focal length $=3 \cdot 0$ $\mathrm{mm}$ to $60 \mathrm{~mm}$; pixel size (still and video modes) $=0.0012 \mathrm{~mm}$; frame rate $=30 \mathrm{~Hz}$ ) were mounted directly underneath an AMTI
OR6-GT-1K glass-topped force plate mounted flush with the walking surface. Synchronisation with the cameras was achieved using a genlocked LED device. A photogrammetric control frame was installed under the force plate to provide object-space control for camera station resection and scaling. The relative coordinates of the targets on the control frame were calculated by a photogrammetric bundle adjustment technique and the detail is provided in Al-Baghdadi et al. (2012). The precision of the relative $\mathrm{x}$ and $\mathrm{y}$ coordinates was $0.025 \mathrm{~mm}$ while the $\mathrm{z}$ coordinate was $0.045 \mathrm{~mm}$.

Image frames from the video clips were extracted using VirtualDub freeware and AVS4YOU proprietary software (Online Media Technologies Ltd, London, UK). Offthe-shelf multi-image bundle adjustment software, Australis (Photometrix, Australia), was utilised in the camcorder calibration, force-plate glass-top refraction distortion calculation, and photogrammetric control calibration. PhotoModeler proprietary software (Eos System Inc. Canada) was used to generate a point cloud of the foot while a MATLAB ${ }^{\circledR}$ protocol was developed to model the glass-top refraction distortion characteristics, detect outlier in measurements, and calculate the distortion correction parameters.

Two healthy adult male subjects in their mid-20s were recruited for the exercise. The 
subjects were prepared for the imaging capture by painting the plantar surface with non-toxic "face and body" paint to improve image capture. Retro-reflective targets were attached to the sole and the side of the foot (Figure 1). The subjects were instructed to walk normally on the gait platform.

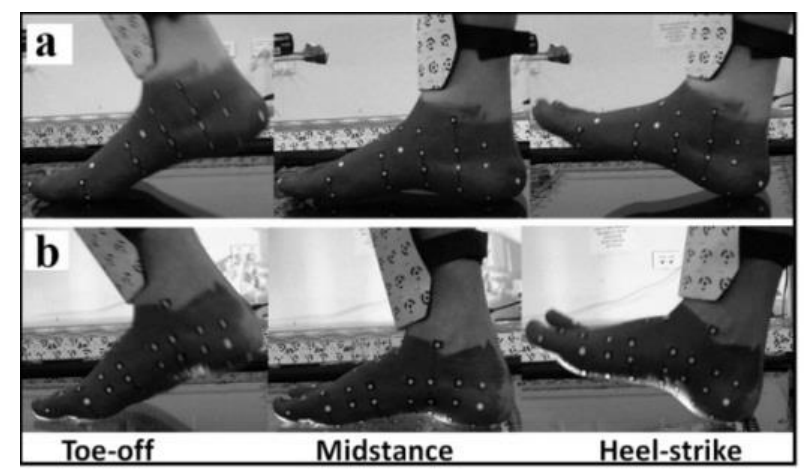

Figure 1 Retro-reflective targets and the gait phase. (a) Subject A. (b) Subject B.

\section{Results}

Figure 2 shows the 3D surface and contours of the plantar surface prior to contact with the force plate (top). The foot arch geometry can be clearly observed from the contour pattern. The lower image of Figure 2 shows the colorized point cloud of the plantar surface during foot strike. The purple color indicates the lowest elevation which is at the same level with the force plate while blue represents the highest elevation. The shape and the distribution of the colors in the point cloud gives the same interpretation of the strike characteristics as shown in Fig 1(b) and shows the heel-strike evenly covers the plantar surface of the heel.
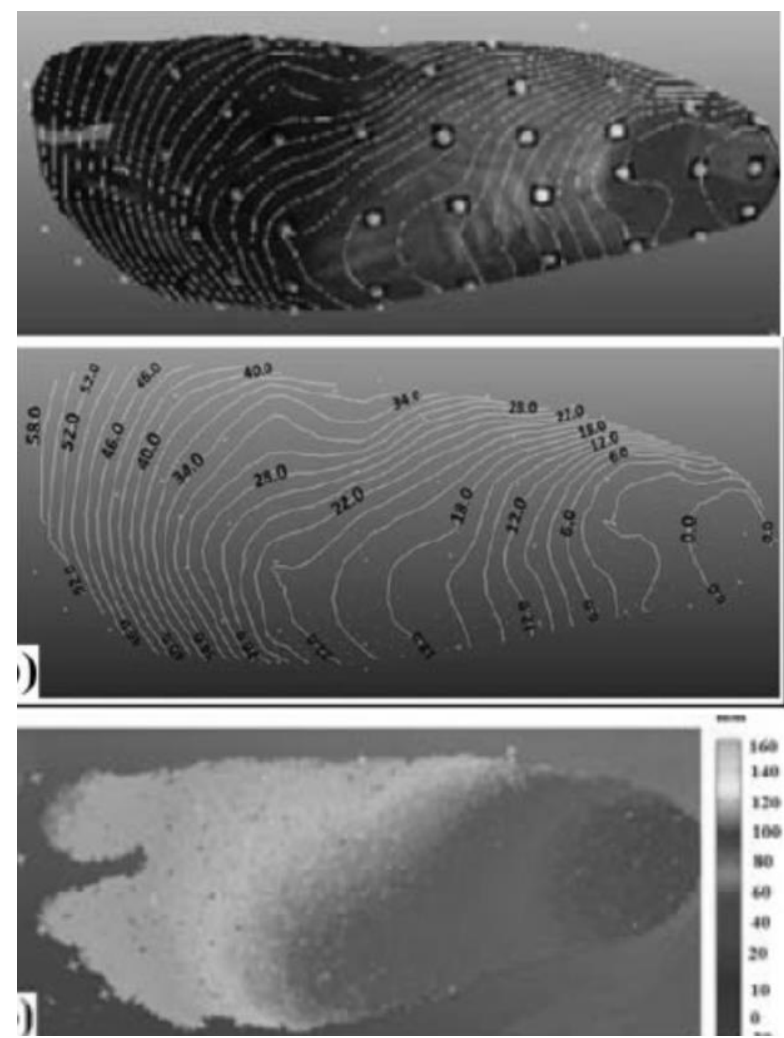

Figure 2 3D plantar surface contours during heel strike.

\section{Discussion and conclusion}

The photogrammetric techniques provide accurate $3 \mathrm{D}$ point clouds for each image frame in the recorded video clips. The calculated 3D surface model can be analyzed using both longitudinal profiles and lateral cross sections to show dynamic characteristics of the plantar surface during the support phase of gait. This capability has not been previously available and provides a better understanding of the plantar soft tissue mechanics and the nature of foot-surface interactions.

\section{References}

Al-Baghdadi, JAA, et al, (2013) Photogramm

Rec. Accepted for publication.

Al-Baghdadi, JAA et al (2012) Proc Int

Workshop Geoinfo Adv. 26-46. 
